\title{
Transforming Acidic Coiled-coil-containing Protein 2 (TACC2) in Human Breast Cancer, Expression Pattern and Clinical/Prognostic Relevance
}

\author{
SHAN CHENG ${ }^{1,2}$, ANTHONY DOUGLAS-JONES ${ }^{3}$, XIAOMEI YANG ${ }^{2}$, \\ ROBERT E. MANSEL ${ }^{1}$ and WEN G. JIANG ${ }^{1}$ \\ ${ }^{1}$ Metastasis and Angiogenesis Research Group, Department of Surgery, and \\ ${ }^{3}$ Department of Pathology, Cardiff University School of Medicine, Heath Park, Cardiff, CF14 4XN, U.K.; \\ ${ }^{2}$ Department of Biochemistry and Molecular Biology, School of Basic Medicine, \\ Capital Medical University, Beijing, P.R. China
}

\begin{abstract}
TACC2 is a member of the transforming acidic coiled-coil-containing protein family and is associated with the centrosome-spindle apparatus during cell cycling. The TACC2 gene is expressed in various splice forms predominantly in postmitotic tissues, including heart, muscle, kidney, and brain. Recent work has shown that members of this family, including TACC2, may be involved in the progression of certain solid tumours. The aim of the current study was to identify the role of TACC2 in breast cancer and to establish if a prognostic relevance existed. Fresh frozen primary human breast cancer tissues $(n=127)$ and nonneoplastic mammary tissues $(n=33)$ were used. The distribution and location of TACC2 was assessed using immunohistochemical staining (IHC). The transcript levels of TACC2 were determined using quantitative real-time PCR. The results were analyzed against the clinical, pathological and follow-up (10 years) data. Statistical analysis was by two-sample t-test and Kaplan-Meier method. TACC2 protein staining was seen in both normal epithelial cells and cancer cells in mammary tissues. Quantitative analysis of the TACC2 transcript revealed a higher level of expression in tumours compared with normal tissues. TACC2 expression was significantly increased in higher grade tumours compared to that in lower grade tumours (grade 3 vs. grade 1, $p=0.046)$. Using the Nottingham Prognostic Index (NPI), TACC2 transcript was significantly higher in tumours from
\end{abstract}

Correspondence to: Professor Wen G. Jiang, Metastasis and Angiogenesis Research Group, University Department of Surgery, Cardiff University School of Medicine, Heath Park, Cardiff CF14 4XN, U.K. Tel: +44 2920742895, Fax: +44 2920742896, e-mail: jiangw@cf.ac.uk

Key Words: TACC2, breast cancer, transcript analysis, survival, prognosis. patients with a moderate prognosis than from those with a good prognosis $(p=0.045)$. The expression in samples from patients with poor clinical outcome (with metastasis, recurrence and breast cancer related death) was higher than that in those from patients who remained disease free. This was reflected by a shorter disease-free survival for patients with high TACC2 (107 (95\% confidence interval: 91-122.8) months compared with 137 (125-150.6) months for patients with low TACC2 transcripts $(p=0.019)$. This study shows that increased expression of TACC2 correlates with poor prognosis in patients with breast cancer. This suggests that TACC2 may mediate an oncogenic effect on breast cancer cells and indicates that TACC2 may be a potential therapeutic target.

The mitotic cell cycle is a precisely controlled process that includes the formation, function, and dissolution of the mitotic spindle. Members of the transforming acidic coiled coil-containing (TACC) family of proteins have been shown to an essential part of this process: a component of the centrosome-spindle apparatus (1). The TACC family is characterized by a highly conserved coiled-coil domain that is essential for localization to the centrosome-spindle apparatus. There is a single TACC gene in Drosophila melanogaster, $A T A C C$, that is hypothesized to play an essential role in stabilizing the centrosome-spindle structure $(2,3)$. Localization to the spindle requires the Drosophila aurora A kinase (4), a kinase required for centrosome maturation (5). Mutants lacking dTACC have abnormally short and weak spindle and astral microtubules, a condition leading to chromosomal missegregation. A Xenopus laevis TACC family member, termed Maskin, has been shown to be involved in sequestering of mRNA complexes to the spindle and suppression of their translation (6-8). This process is mediated through the association of Maskin with a cytoplasmic polyadenylation element-binding protein and 
with eIF4E. Cells injected with antibodies against Maskin display mitotic defects including disruption of the centrosome-spindle apparatus. More recently, a distantly related TACC protein was identified in Caenorhabditis elegans, TAC-1 $(9,10)$, which has been shown to regulate the growth and assembly of microtubules.

The mammalian TACC proteins (TACC1, TACC2, and TACC3) have only recently been identified. The functions of the three known mammalian TACC proteins are largely unknown, but several observations suggest that the proteins may contribute to cancer. For example, the genes encoding TACC proteins are all in genomic regions that are rearranged in certain types of cancer. TACC1 and TACC2 are located on chromosomes 8p11 and 10q26, respectively, two regions that are implicated in breast cancer and other tumours (11). TACC3 was mapped to 4p16, within a translocation breakpoint region associated with the disease multiple myeloma (12). However, the role of the TACC family in the development and progression of cancer is controversial. TACC1, the first family member identified, was discovered as the product of a gene that is amplified in breast cancer (11). The expression of specific TACC1 isoforms was also implicated in the development of gastric (13) and prostate cancer (14). On the other hand, TACC1 protein had been shown to be significantly reduced in $50 \%$ of resected breast tumours compared to normal levels, suggesting that TACC1 may be a breast tumour suppressor gene (15). TACC3 is also up-regulated in several cancer cell lines, including lung cancer $(12,16)$; yet, again, it was reported as being absent or reduced in ovarian and thyroid cancer tissues (17). Initially, it was suggested that the TACC2 splice variant AZU-1 is a tumour suppressor in breast cancer. Over-expression of the short isoform, TACC 2 s, can reduce the in vitro tumourigenic properties of oestrogen receptor-negative breast cancer cell lines (18). However, the lack of any tumour phenotype in TACC2knockout mice did not support this idea (19). It therefore appears that these proteins can be up- or down-regulated in different types of cancer or, surprisingly, even in the same type $(13,15,16,17,19-23)$; as such, their putative involvement in cancer development and/or progression remains unclear.

The aim of the current study was to further identify the role of TACC2 in breast cancer and to establish if a prognostic relevance exists.

\section{Materials and Methods}

Human breast specimens. A total of 160 breast samples were obtained from breast cancer patients (33 were background normal breast tissue and 127 breast cancer tissue). These tissues were collected immediately after mastectomy, and snap-frozen in liquid nitrogen, with the approval of the Local Ethical Committee. Normal background mammary tissues were removed from the same patients.
Table I. Breast cancer patient clinical data details.

\begin{tabular}{|c|c|c|}
\hline Clinical data & Grouping & Sample number \\
\hline \multirow[t]{2}{*}{ Tissue sample } & Background & 33 \\
\hline & Tumour & 127 \\
\hline \multirow[t]{3}{*}{ Nottingham Prognostic Index } & $1(<3.4)$ & 72 \\
\hline & $2(3.4-5.4)$ & 39 \\
\hline & $3(>5.4)$ & 16 \\
\hline \multirow[t]{3}{*}{ Tumour grade } & 1 & 24 \\
\hline & 2 & 44 \\
\hline & 3 & 59 \\
\hline \multirow[t]{4}{*}{ TNM staging } & 1 & 73 \\
\hline & 2 & 42 \\
\hline & 3 & 8 \\
\hline & 4 & 4 \\
\hline \multirow[t]{4}{*}{ Survival status } & 1 & 92 \\
\hline & 2 & 13 \\
\hline & 3 & 6 \\
\hline & 4 & 16 \\
\hline
\end{tabular}

The pathologist verified normal background and cancer specimens, and confirmed that the background samples were free from tumour deposits. Tumour samples were macro-dissected by the pathologist. The follow-up for the cohort was 10 years. For patient clinical data see Table I.

Real-time quantitative polymerase chain reaction ( $Q P C R)$. The iCycler IQ system (BioRad, Camberley, UK) was employed to quantify the level of TACC2 transcript in the breast specimens (shown as copies 50 ng RNA from internal standard). Breast cDNA samples were then examined for TACC2 transcript expression, along with a set of standards and negative controls (TACC2 QPCR primers: sense: CCCCAACAATCATACAACTT; antisense: ACTGAACCTGACCGT ACATGGGGATTTAGAAGGTGAG). The QPCR technique utilized the Amplifluor ${ }^{\mathrm{TM}}$ system (Intergen Inc., UK) and QPCR master mix (ABgene, Surrey, UK), in conjunction with a universal probe (UniPrimer ${ }^{\mathrm{TM}}$ ), as previously reported (24-26). Real-time QPCR conditions were $95^{\circ} \mathrm{C}$ for $15 \mathrm{~min}$, followed by 60 cycles at $95^{\circ} \mathrm{C}$ for $20 \mathrm{~s}, 55^{\circ} \mathrm{C}$ for $30 \mathrm{~s}$ and $72^{\circ} \mathrm{C}$ for $20 \mathrm{~s}$. The results of the test molecules were normalised against levels of $\beta$-actin using a $\beta$-actin quantification kit from Perkin-Elmer (Perkin-Elmer, Surrey, UK).

Immunohistochemical staining of breast specimens. This was based on methods recently reported (27). Frozen sections of breast tumour $(n=20)$ and background tissue $(n=20)$ were cut at a thickness of $6 \mu \mathrm{m}$ using a cryostat. The sections were mounted on super frost plus microscope slides, air-dried and then fixed in a mixture of $50 \%$ acetone and $50 \%$ methanol. The sections were then placed in 'Optimax' wash buffer for 5-10 min to rehydrate. Sections were incubated for $20 \mathrm{~min}$ in a horse serum blocking solution and probed with the TACC2 antibody (1:200) (SantaCruz Biotechnologies, California, USA). This study employed controls that omitted the primary and secondary antibodies. 


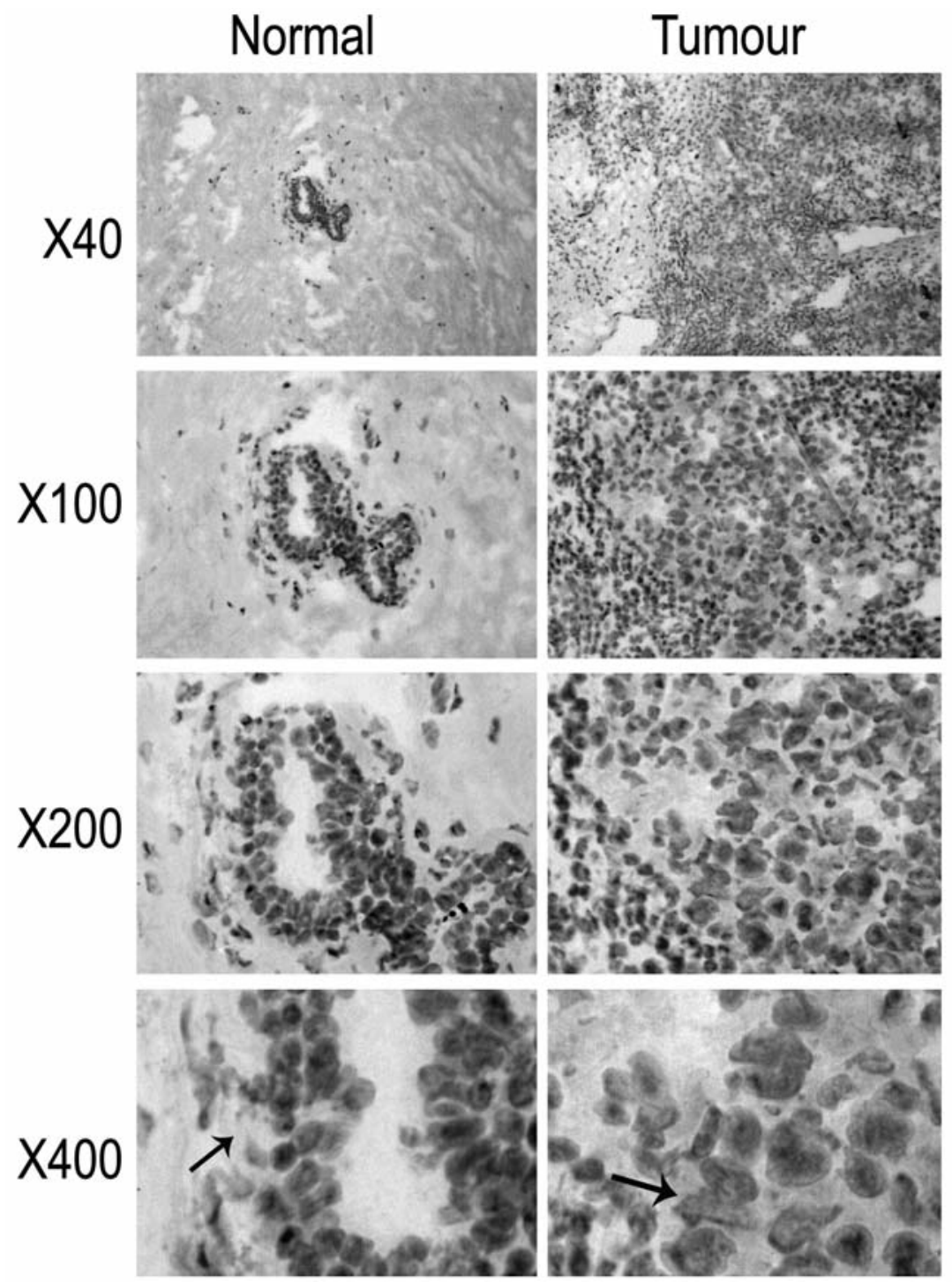

Figure 1. Immunohistochemical staining of mammary tissues. Left panel: an example of normal background breast tissue. TACC2 was weakly expressed in the normal mammary tissue (black arrows in right panel) and its distribution was confined to the ductal epithelium. Right panel: breast cancer tissue. Staining for TACC2 was seen to be strongly positive ithroughout the breast tumour cells (black arrows in right panel), when compared against the intense epithelial staining of the normal breast tissues.

Furthermore, control tests were also conducted using purified IgG from non-immunised sheep (Sigma, Poole, Dorset, UK). Following extensive washings, sections were incubated for $30 \mathrm{~min}$ in the secondary biotinylated antibody (1:100) (Multilink Swine Anti-goat/mouse/rabbit Immunoglobulin, Dako Inc). Following washings, Avidin Biotin Complex (Vector Laboratories, Peterborough, UK) was then applied to the sections, followed by extensive washing steps. Diaminobenzidine chromogen (Vector Labs, Peterborough, UK) was then added to the sections, and incubated in the dark for $5 \mathrm{~min}$. Sections were then dehydrated in ascending grades of methanol before clearing in xylene and mounting under a coverslip. Staining was independently assessed by the Authors. Breast tumour tissue was also stained in the absence of TACC 2 primary antibody to act as a negative control.
Statistical analysis. Statistical analysis was performed using Minitab ${ }^{\circledR}$, version 14 (State College, PA, USA). Two-sample $t$-test was used for normally distributed data. Survival analysis was carried out using the Kaplan-Meier method on SPSS (version 15, Chicago, IL, USA). Differences were considered statistically significant at $p<0.05$.

\section{Results}

Immunohistochemical staining of TACC2 protein in human mammary tissues. TACC2 was stained in the normal mammary tissue (Figure 1, left panels). Its distribution was mostly confined to the ductal epithelial cells. Limited staining was seen in myoepithelial cells and stromal 

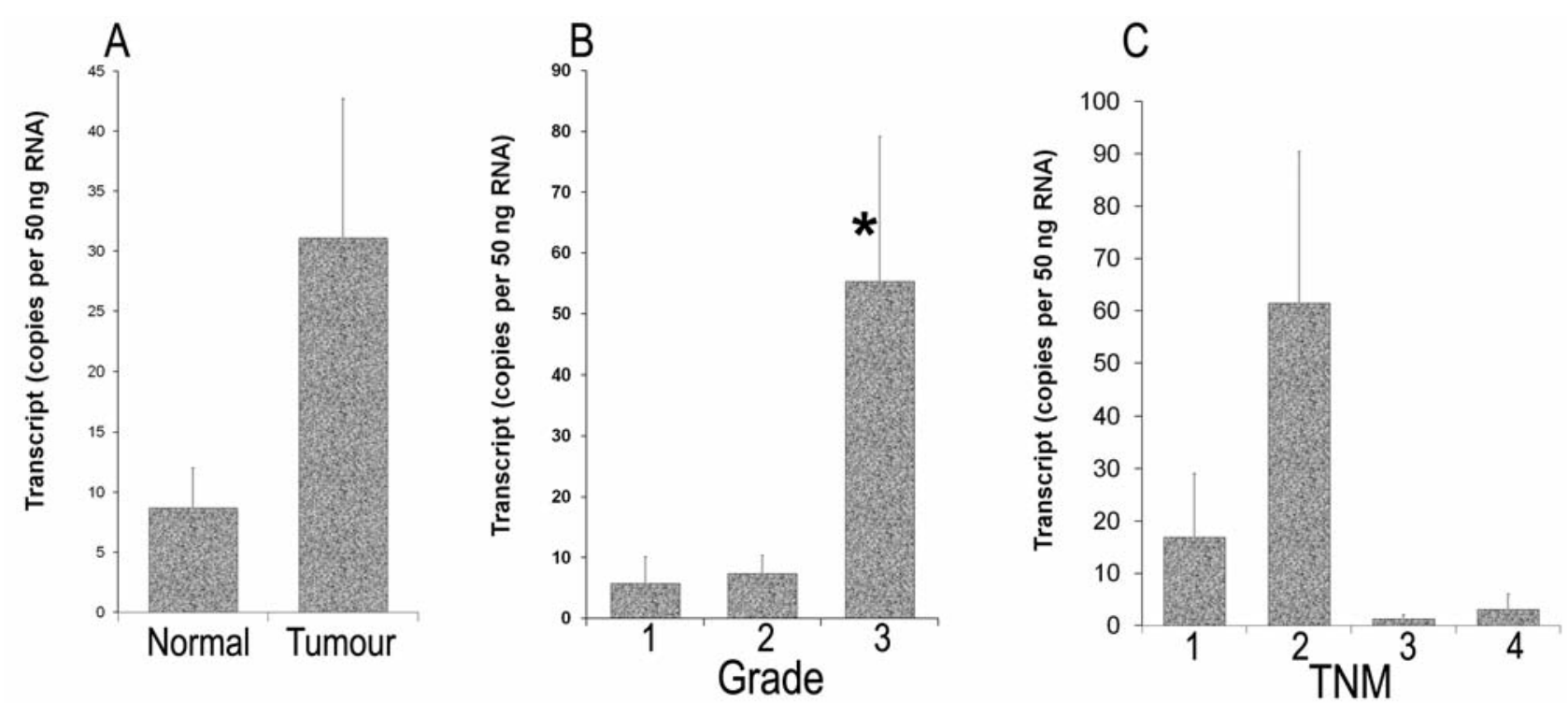

Figure 2. Quantitative-PCR analysis of TACC2 expression levels in breast cancer patient specimens. A: Tumour versus background tissue from breast cancer patients. Expression according to: B: tumour grade; * $p=0.046 \mathrm{vs}$ grade-1; C: tumour-node-metastasis classification of patients.

fibroblasts. In tumour tissues, TACC2 protein was also seen, again primarily in breast cancer cells (Figure 1, right panels).

Quantitative-PCR analysis of TACC2 transcripts in mammary tissues. Tumour tissues $(31.1 \pm 11.6)$ had increased levels of TACC 2 compared to normal background tissues $(8.69 \pm 3.36)$, although these values did not reach statistical significance (Figure 2A).

TACC 2 expression in relation to tumour grade. Grade 3 tumours (55.3 \pm 23.9$)$ had significantly increased levels of TACC2, when compared to the TACC2 levels in well differentiated grade 1 tumours, $p=0.046$ (Figure 2B). There was a trend that suggests grade 2 tumours $(7.37 \pm 3.01)$ had increased levels of TACC2 compared to grade 1 tumours $(5.79 \pm 4.39)$.

TACC2 and tumour staging. We also analysed TACC2 expression in relation to TNM staging, classified after surgery (Figure 2C). TNM2 $(61.5 \pm 28.9)$ tumours had a higher level of TACC2 than TNM1 tumours (16.9 \pm 12.2$)$, although the difference was not statistically significant. There is no significant difference between TNM3 (1.302 \pm 0.788$)$, TNM4 (3.15 \pm 2.9$)$ and TNM1 tumours.

TACC2 expression in relation to patient prognosis indices. The levels of TACC2 transcripts were analysed against the Nottingham Prognostic Index (NPI) (Figure 3A), which divided the patients into three groups: the NPI1 group (NPI score <3.4) represented patients with a good prognosis; the NPI2 group (NPI score 3.4-5.4) represented patients with a moderate prognosis; whereas the NPI3 patients (NPI score >5.4) had a poor prognosis. Tumours from patients with moderate and poor prognoses had increased levels of TACC2 (73.6 \pm 35.3 and $29.8 \pm 12.8$, respectively) when compared with those from patients of good prognosis group (7.2 \pm 2.78 ). TACC2 transcript was significantly higher in tumours from patients with a moderate prognosis than from those with a good prognosis $(p=0.045)$ (Figure $3 \mathrm{~A})$.

TACC2 expression, clinical outcome and long-term survival. Patients were divided into four subgroups with a 10-year median follow-up period: those patients who were alive, well and free of recurrence; patients with local recurrence; patients with distant metastasis; and patients who had died as a result of breast cancer. We report that patients who had died of breast cancer $(102.5 \pm 65.3)$ and patients with metastatic disease $(54.1 \pm 33.5)$ had significantly higher levels

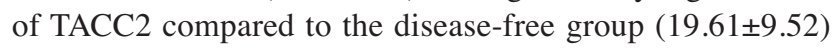
although this observation was not statistically significant (Figure 3B). Using the Kaplan-Meier survival curve method and Cox proportion hazards analysis, it was revealed that the patients with low levels of TACC2 expression in their tumours showed significantly longer survival of 137 (125150.6) months than those with higher expression levels (107 (91-122.8, 95\% CI) months $(p=0.019)$ (Figure 3D). Furthermore, comparison of TACC2 levels and overall patient survival (Figure 3C) also revealed that patients with low TACC2 levels had a longer overall survival compared to those whose tumours expressed high levels of TACC2, although the difference did not reach statistical significance. 

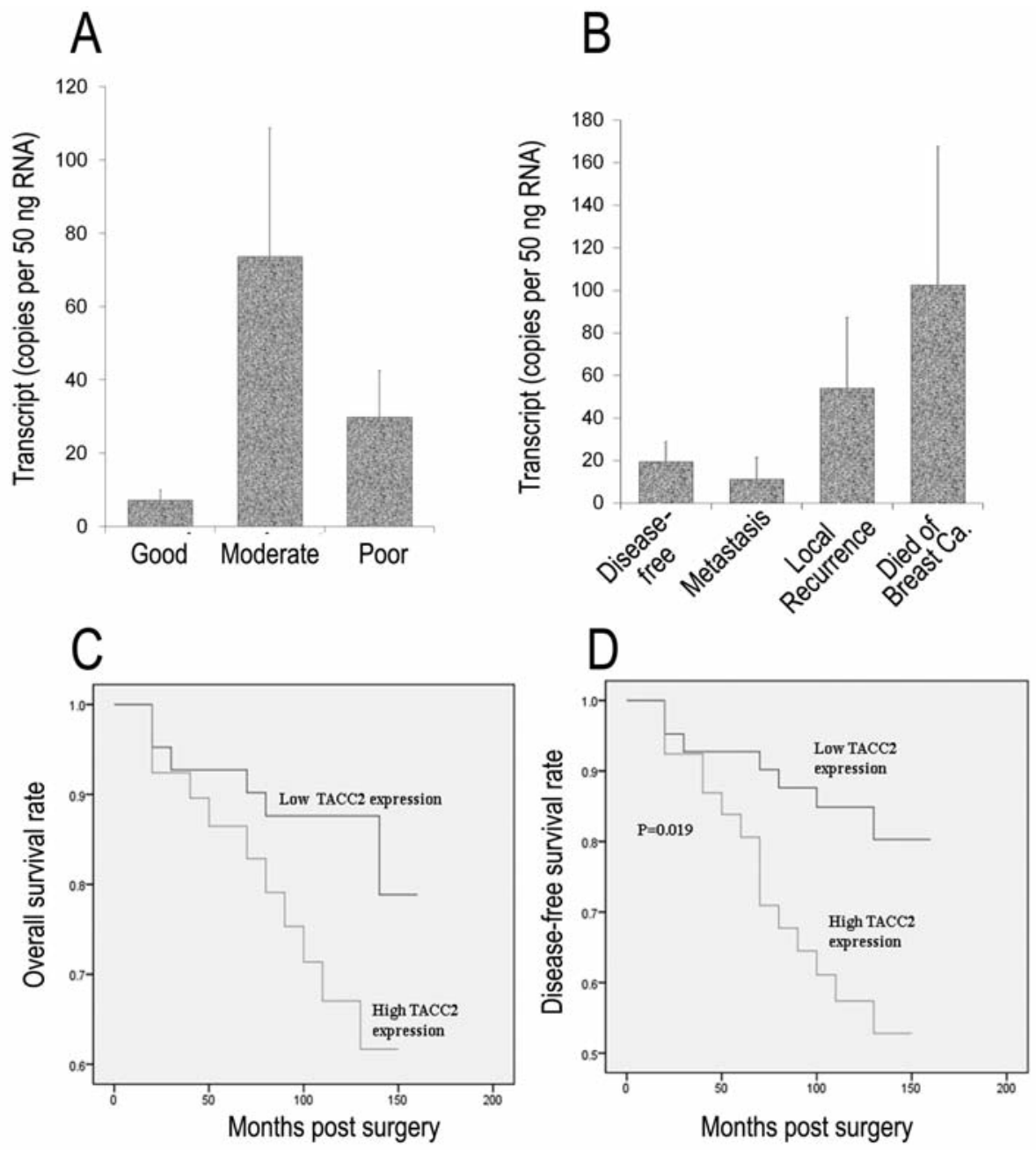

Figure 3. TACC2 transcript level in relation to prognosis and clinical outcome. A: Nottingham Prognostic Index of patients. Good, moderate and poor prognostic groups had NPI indices <3.4, 3.4-5.4 and >5.4, respectively. B: Clinical outcome after a 10-year followup. C: Kaplan-Meier overall survival analysis. D: Kaplan-Meier disease-free survival analysis.

\section{Discussion}

The mammalian family of TACC proteins consists of TACC1, TACC2, and TACC3. Interestingly, each of the TACC family member genes chromosomally co-localizes with one of the fibroblast growth factor receptor genes (12) and, while there are four fibroblast growth factor receptor genes, a fourth TACC gene has yet to be identified. As illustrated in previous studies, each of the TACC family members has a unique pattern of expression that only partially overlaps with those of other family members.

The three human genes encoding TACC proteins are all in genomic regions that are rearranged in certain types of cancer, and their expression is altered in tumours from different tissues. TACC1, the first family member identified, was discovered as the product of a gene that is amplified in breast cancer (11) and more recently was also shown to be overexpressed in prostate cancer (14). TACC3 was identified independently as an Ah receptor nuclear translocator protein-interacting protein and a putative player in hypoxic responses (28), the product of an erythropoietin-induced gene in hematopoietic progenitors (29), and a Stat5-interacting protein in a yeast two-hybrid screen (30). TACC2 was identified independently in several laboratories and has been hypothesized to have several different biological functions. In one laboratory (22), a splice variant of the TACC2 gene was proposed to be a breast cancer tumour suppressor gene based on the observations that it was downregulated in certain malignant cell clones and that when overexpressed in cell lines, it could suppress cell growth and metastatic properties (22). The TACC2 gene was also identified as a gene that is induced in cultured microvascular endothelial cells in response to erythropoietin (31) and proposed to be an 
important component in the control of blood vessel growth and development. The detection of interaction of TACC2 with the nuclear histone acetyltransferase hGCN5L2 led to the suggestion that it may function in the regulation of gene transcription (32), although TACC2 was suggested to form other complexes (33). It has also been considered that TACC2 lacks any critical, nonredundant functions due to a lack of any phenotypic changes when the TACC2 gene was deleted in mice (19).

Although TACC2 has been suggested to be a possible metastasis suppressor, there is little evidence for this. The present study indicates that TACC2 may be an indicator of survival of patients with breast cancer, as patients with a poor prognosis expressed elevated levels of TACC2.TACC2 mRNA levels were dramatically increased in patients who died of breast cancer compared with patients who were alive and well. Our survival analysis also shows that over a 10-year follow-up, patients whose tumours expressed low levels of TACC2 were statistically more likely to survive, cancer-free, for longer than those patients with a high TACC2 level. These results demonstrate that the degree of TACC 2 expression may be relevant in terms of breast cancer patient survival. In view of our data, we suggest that TACC2 acts as an indicator for breast cancer patients, in that patients expressing low/reduced levels of TACC2 have a poor prognosis, in contrast to those patients with high/elevated levels of TACC2 and a favorable prognosis. Although several observations indicated a contrasting role for TACC 2 protein in processes underlying the development of cancer, our study provides new evidence that $T A C C 2$ may demonstrate an oncogenic role in modulating metastatic ability in breast cancer.

In conclusion, our study shows that TACC2 demonstrates the ability to modulate metastatic ability in breast cancer cells. We also report that TACC2 expression levels may act as an indicator of patient survival. Therefore, TACC2 has prognostic value for breast cancer patients.

\section{Acknowledgements}

We wish to thank Cancer Research Wales, the Albert Hung Foundation, National Natural Science Foundation of the People's Republic of China (No. 30800187) and New Star Plan of Science and Technology of Beijing of China (No. 2007B065) for funding this research.

\section{References}

1 Gergely F: Centrosomal TACCtics. Bioessays 24: 915-925, 2002.

2 Gergely F, Kidd K, Jeffers D, Wakefield JG and Raff JW: D-TACC: a novel centrosomal protein required for normal spindle function in the early Drosophila embryo. EMBO J 19: 241-252, 2000.

3 Lee MJ, Gergely F, Jeffers K, Peak-Chew SY and Raff JW: Msps/XMAP215 interacts with the centrosomal protein D-TACC to regulate microtubule behaviour. Nat Cell Biol 3: 643-649, 2001.
4 Giet R, McLean D, Descamps S, Lee MJ, Raff JW, Prigent C and Glover DM: Drosophila aurora A kinase is required to localize D-TACC to centrosomes and to regulate astral microtubules. J Cell Biol 156: 437-451, 2002.

5 Hannak E, Kirkham M, Hyman AA and Oegema K: Aurora-A kinase is required for centrosome maturation in Caenorhabditis elegans. J Cell Biol 155: 1109-1116, 2001.

6 Cao Q and Richter JD: Dissolution of the maskin-eIF4E complex by cytoplasmic polyadenylation and poly(A)-binding protein controls cyclin B1 mRNA translation and oocyte maturation. EMBO J 21: 3852-3862, 2002.

7 Groisman I, Huang YS, Mendez R, Cao Q, Theurkauf W and Richter JD: $C P E B$, maskin, and cyclin B1 mRNA at the mitotic apparatus: implications for local translational control of cell division. Cell 103: 435-447, 2000.

8 Stebbins-Boaz B, Cao Q, de Moor CH, Mendez R and Richter JD: Maskin is a CPEB-associated factor that transiently interacts with elF-4E. Mol Cell 4: 1017-1027, 1999.

9 Le Bot N, Tsai MC, Andrews RK and Ahringer J: TAC-1, a regulator of microtubule length in the C. elegans embryo. Curr Biol 13: 1499-1505, 2003.

10 Srayko M, Quintin S, Schwager A and Hyman AA: Caenorhabditis elegans TAC-1 and ZYG-9 form a complex that is essential for long astral and spindle microtubules. Curr Biol 13: 1506-1511, 2003.

11 Still IH, Hamilton M, Vince P, Wolfman A and Cowell JK: Cloning of TACC1, an embryonically expressed, potentially transforming coiled-coil-containing gene, from the $8 \mathrm{p} 11$ breast cancer amplicon. Oncogene 18: 4032-4038, 1999.

12 Still IH, Vince P and Cowell JK: The third member of the transforming acidic coiled-coil-containing gene family, TACC3, maps in 4 p 16 , close to translocation breakpoints in multiple myeloma, and is upregulated in various cancer cell lines. Genomics 58: 165-170, 1999.

13 Line A, Slucka Z, Stengrevics A, Li G and Rees RC: Altered splicing pattern of TACC1 mRNA in gastric cancer. Cancer Genet Cytogenet 139: 78-83, 2002.

14 Dhanasekaran SM, Barrette TR, Ghosh D, Shah R, Varambally S, Kurachi K, Pienta KJ, Rubin MA and Chinnaiyan AM: Delineation of prognostic biomarkers in prostate cancer. Nature 412: 822-826, 2001.

15 Conte N, Delaval B, Ginestier C, Ferrand A, Isnardon D, Larroque C, Prigent C, Séraphin B, Jacquemier J and Birnbaum D: TACC1-chTOG-Aurora A protein complex in breast cancer. Oncogene 22: 8102-8116, 2003.

16 Jung CK, Jung JH, Park GS, Lee A, Kang CS and Lee KY: Expression of transforming acidic coiled-coil containing protein 3 is a novel independent prognostic marker in non-small cell lung cancer. Pathol Int 56: 503-509, 2006.

17 Ulisse S, Baldini E, Toller M, Delcros JG, Guého A, Curcio F, De Antoni E, Giacomelli L, Ambesi-Impiombato FS, Bocchini S, D'Armiento M and Arlot-Bonnemains Y: Transforming acidic coiled-coil 3 and Aurora-A interact in human thyrocytes and their expression is deregulated in thyroid cancer tissues. Endocr Relat Cancer 14: 827-837, 2007.

18 Lauffart B, Gangisetty O and Still IH: Molecular cloning, genomic structure and interactions of the putative breast tumour suppressor TACC2. Genomics 81: 192-201, 2003.

19 Schuendeln MM, Piekorz RP, Wichmann C, Lee Y, McKinnon PJ, Boyd K, Takahashi Y and Ihle JN: The centrosomal, putative 
tumour suppressor protein TACC2 is dispensable for normal development, and deficiency does not lead to cancer. Mol Cell Biol 24: 6403-6409, 2004.

20 Lauffart B, Vaughan MM, Eddy R, Chervinsky D, DiCioccio RA, Black JD and Still IH: Aberrations of TACC1 and TACC3 are associated with ovarian cancer. BMC Womens Health 5: 8, 2005.

21 Raff JW: Centrosomes and cancer: lessons from a TACC. Trends Cell Biol 12: 222-225, 2002.

22 Chen HM, Schmeichel KL, Mian IS, Lelièvre S, Petersen OW and Bissell MJ: AZU-1: a candidate breast tumour suppressor and biomarker for tumour progression. Mol Biol Cell 11: 1357 1367, 2000.

23 Conte N, Charafe-Jauffret E, Delaval B, Adélaïde J, Ginestier C, Geneix $\mathrm{J}$, Isnardon $\mathrm{D}$, Jacquemier $\mathrm{J}$ and Birnbaum $\mathrm{D}$ : Carcinogenesis and translational controls: TACC1 is downregulated in human cancers and associates with mRNA regulators. Oncogene 21: 5619-5630, 2002.

24 Nazarenko IA, Bhatnagar SK and Hohman RJ: A closed tube format for amplification and detection of DNA based on energy transfer. Nucleic Acids Res 25: 2516-2521, 1997.

25 Bokobza SM, Ye L, Kynaston H, Mansel RE and Jiang WG: Reduced expression of BMPR-IB correlates with poor prognosis and increased proliferation of breast cancer cells. Cancer Genomics Proteomics 6: 101-108, 2009.

26 Jiang WG, Martin TA, Russell-Lewis J, Ye L, Douglas-Jones A and Mansel RE: Eplin-alpha expression In human breast cancer, the impact on cellular migration and clinical outcome. Mol Cancer 7: 71, 2008.

27 Jiang WG, Davies G, Martin TA, Parcc C, Watkins G, Mason MD, Mokbel $\mathrm{K}$ and Mansel RE: Molecular targeting of matrilysin and its impact on tumour growth in vivo, the potential implications in breast cancer therapy. Clin Cancer Res 11: 60126019, 2005.
28 Sadek CM, Jalaguier S, Feeney EP, Aitola M, Damdimopoulos AE, Pelto-Huikko $\mathrm{M}$ and Gustafsson JA: Isolation and characterization of AINT: a novel ARNT interacting protein expressed during murine embryonic development. Mech Dev 97: 13-26, 2000.

29 McKeveney PJ, Hodges VM, Mullan RN, Maxwell P, Simpson D, Thompson A, Winter PC, Lappin TR and Maxwell AP: Characterization and localization of expression of an erythropoietin-induced gene, ERIC-1/TACC3, identified in erythroid precursor cells. Br J Haematol 112: 1016-1024, 2001.

30 Piekorz RP, Hoffmeyer A, Duntsch CD, McKay C, Nakajima H, Sexl V, Snyder L, Rehg J and Ihle JN: The centrosomal protein TACC 3 is essential for hematopoietic stem cell function and genetically interfaces with p53-regulated apoptosis. EMBO J 21: 653-664, 2002.

$31 \mathrm{Pu} \mathrm{JJ}, \mathrm{Li} \mathrm{C}$, Rodriguez $\mathrm{M}$ and Banerjee D: Cloning and structural characterization of ECTACC, a new member of the transforming acidic coiled-coil (TACC) gene family: cDNA sequence and expression analysis in human microvascular endothelial cells. Cytokine 13: 129-137, 2001.

32 Gangisetty O, Lauffart B, Sondarva GV, Chelsea DM and Still IH: The transforming acidic coiled-coil proteins interact with nuclear histone acetyltransferases. Oncogene 23: 2559-2563, 2004.

33 Lauffart B, Howell SJ, Tasch JE, Cowell JK and Still IH: Interaction of the transforming acidic coiled-coil 1 (TACC1) protein with ch-TOG and GAS41/NuBI1 suggests multiple TACC1-containing protein complexes in human cells. Biochem $\mathrm{J}$ 363: 195-200, 2002.

Received February 7, 2010 Accepted February 16, 2010 